\title{
SIMULATION OF ULTRASONIC IMAGING WITH LINEAR ARRAYS IN CAUSAL ABSORPTIVE MEDIA
}

\author{
A. P. Berkhoff, J. M. Thussen and R. J. F. Homan \\ Biophysics Laboratory, Department of Ophthalmology, St. Radboud University Hospital, \\ Nijmegen, The Netherlands
}

(Received 3 April 1995; in final form 26 July 1995)

\begin{abstract}
Rigorous and efficient numerical methods are presented for simulation of acoustic propagation in a medium where the absorption is described by relaxation processes. It is shown how FF" $\mathrm{T}$-based algorithms can be used to simulate ultrasound images in pulse-echo mode. General expressions are obtained for the complex wavenumber in a relaxing medium. A fit to measurements in biological media shows the appropriateness of the model. The wavenumber is applied to three FFT-based extrapolation operators, which are implemented in a weak form to reduce spatial aliasing. The influence of the absorptive medium on the quality of images obtained with a linear array transducer is demonstrated. It is shown that, for moderately absorbing media, the absorption has a large influence on the images, whereas the dispersion has a negligible effect on the images.
\end{abstract}

Key Words: Ultrasound simulation, Causal absorptive media, Wavefield extrapolation, Plane-wave expansion, Linear array, Dispersion, Image quality.

\section{INTRODUCTION}

Accurate and efficient numerical methods can be very useful for studying transducer performance in ultrasonic imaging applications. Especially for absorbing media and complex transducer geometries, the tradeoff between pulse shape, resolution and penetration cannot always be predicted by approximate calculation rodels.

Surprisingly few reports deal with the rigorous simulation of ultrasonic propagation in absorptive media. To our knowledge, we are the first to combine a model based wavenumber with rigorous field calculation methods in ultrasound pulse-echo imaging for multielement transducers. Christopher and Parker ( 991$)$ employ a power-law absorption for their numerical wave propagation methods. Pulse-echo behavicur is studied in a rigorous way by the finite element iraplementation of Lerch and Landes (1994). Simulation methods for pulse-echo behaviour are also presented by Pedersen and Orofino (1994) and Orofino and Pedersen (1993). The latter three articles have in common that no images are shown. Simulated images

Address correspondence to: J. M. Thijssen, Ph.D., Clinical Physics Laboratory, University Children's Hospital, St. Radboud University Hospital, P.O. Box 9101, 6500 HB Nijmegen, The Netherlands. have been shown by Foster et al. (1983) and Turnbull et al. (1992), but not for absorptive media. Goodsitt et al. (1983) use three-dimensional (3D) single-element transducer simulations to obtain speckle images in absorptive media, but their article lacks information on the medium model used.

Various models have been proposed for the description of absorption in biological media. Most of the models are based on a power-law description of the absorption versus frequency. However, the physical explanation of these models is not always clear. Noteworthy exceptions include the minimum-phase approaches of Kuc (1984), where the propagation in tissue is described by minimum-phase filters, and Gurumurthy and Arthur (1982), who derived a minimumphase function using the Hilbert transform. General relationships between absorption and phase velocity were given by O'Donnell et al. (1978, 1981). A common opinion in the ultrasound literature (Jongen et al. 1986; Pauly and Schwan 1971; Sehgal and Greenleaf 1982 ) is that the wave propagation in biological media can be described by a continuous spectrum of relaxation processes. According to Dunn et al. (1969), a discrete set of relaxation processes is more acceptable in physical terms than the continuous relaxation spectrum distribution, even in complex situations as in an 
aqueous solution of haemoglobin. However, Bamber (1986) states that it is not known whether a discrete number of processes are involved or whether the distribution of relaxation frequencies is a continuous function of frequency. Our models aim at a description of biological soft tissues in general, where it would be convenient to have the attenuation slope as one of the parameters. The attenuation slope is one of the few parameters that can be used to differentiate tissues in a stable way. It will be seen that the attenuation slope is one of the parameters of our continuous relaxation models.

We present a rigorous method based on monochromatic plane waves, where time-domain results and arbitrary field distributions are obtained by linear superposition. This approach has the advantage that the computation time is not proportional to the number of transducer elements as is the case for most time-domain-based methods (Foster et al. 1983; Turnbull et al. 1992). An outline of the article is as follows: First, the discrete relaxation model from Nachman et al. (1990) will be reviewed. To reduce the number of parameters and improve the numerical behaviour of fitting experimental data, we extend the model to a continuous distribution of relaxation processes in the next section. Subsequently, we will combine methods of $\mathbf{k}$-space wave-field extrapolation with our complex wavenumber for rigorous simulations in absorptive media. To arrive at stable and accurate results, we introduce three weak forms of wave-field extrapolation operators. As an example, an analysis of the images obtaincd with a lincar array transducer is presented. We will show the influence of absorption and dispersion on the image quality.

\section{CAUSAL ABSORPTION MODEL FOR MEDIA DESCRIBED BY RELAXATION PROCESSES}

In this article, it is assumed that the medium consists of a base medium with sound speed, $c_{0}$, and density, $\rho_{0}$. Molecules of different materials are dissolved in the base medium. The dissolved molecules cause a change of the compressibility at higher frequencies. The density is assumed to be the same for the base medium and the dissolved molecules. Our starting point is a model where a discrete set of relaxation processes contributes to the absorption in the medium. The model is very useful for a theoretical description of wave propagation in biological media. However, we encountered numerical problems when obtaining a model fit, even for only three or four discrete relaxation processes. Methods to circumvent this problem are discussed in subsequent sections. Simple analytical results will be given for two cases.

\section{General model}

The results from Nachman et al. (1990) will be briefly reviewed, with emphasis on the frequency domain results. The complex wavenumber, $k(\omega)$, can be described by:

$$
k(\omega)=\omega\left(\frac{1}{c_{0}^{2}}+\rho_{0} \sum_{j^{\prime}=1}^{N} \frac{i \omega \kappa_{\nu}}{-i \omega+\Omega_{\nu}}\right)^{1 / 2}
$$

with $c_{0}$ the sound speed at zero frequency, $\rho_{0}$ the equilibrium density of the medium and $\omega$ the angular frequency. Each relaxation process, $\nu$, is characterized by an effective compressibility, $\kappa_{\nu}$, and an angular relaxation frequency, $\Omega_{\nu}$. The generalized (frequency domain) compressibility can be defined as:

$$
\kappa(\omega)=\frac{1}{c_{0}^{2} \rho_{0}}+\sum_{\nu=1}^{N} \frac{i \omega \kappa_{\nu}}{-i \omega+\Omega_{\nu}}
$$

The zero-frequency compressibility, $\kappa_{0}$, is given by:

$$
\kappa_{0} \triangleq \kappa(0)=\frac{1}{c_{0}^{2} \rho_{0}}
$$

The high-frequency compressibility, $\kappa_{\infty}$, is given by:

$$
\kappa_{\infty} \triangleq \kappa(\infty)=\frac{1}{c_{0}^{2} \rho_{0}}-\sum_{\nu=1}^{N} \kappa_{\nu}
$$

This quantity should be positive. In the lossless case, $\kappa_{0}$ and $\kappa_{\infty}$ are identical. The generalized time-domain compressibility, $\kappa(t)$, can be obtained explicitly by Fourier-transforming $\kappa(\omega)$ :

$$
\kappa(t)=\frac{1}{2 \pi} \int_{-\infty}^{\infty} \kappa(\omega) e^{-i \omega t} d \omega
$$

Using residues we find that the compressibility is identically zero for $t<0$. The nonzero causal part is given by:

$$
\kappa(t)=\kappa_{\infty} \delta(t)+\sum_{\nu=1}^{N} \Omega_{\nu} \kappa_{\nu} e^{-\Omega_{\nu} t}, \quad t \geq 0
$$

If we write:

$$
\mathrm{k}(\omega)=\frac{\omega}{c(\omega)}+i \alpha(\omega)
$$

and use the relation for the frequency-dependent compressibility, we can determine the frequency-dependent 
(dispersive) velocity, $c(\omega)$, and the frequency-dependent attenuation coefficient, $\alpha(\omega)$ :

$$
\begin{aligned}
c(\omega)= & \left(\frac { \rho _ { 0 } } { 2 } \left\{\kappa_{\infty}+\lambda(\omega)\right.\right. \\
& \left.\left.+\left[\left(\kappa_{\infty}+\lambda(\omega)\right)^{2}+\mu^{2}(\omega)\right]^{1 / 2}\right\}\right)^{-1 / 2} \\
\alpha(\omega)=\omega\left(\frac { \rho _ { 0 } } { 2 } \left\{-\kappa_{\infty}-\lambda(\omega)\right.\right. & \\
& \left.\left.+\left[\left(\kappa_{\infty}+\lambda(\omega)\right)^{2}+\mu^{2}(\omega)\right]^{1 / 2}\right\}\right)^{1 / 2}
\end{aligned}
$$

with:

$$
\lambda(\omega)=\sum_{j=1}^{N} \frac{\kappa_{\nu}}{1+\left(\omega / \Omega_{\nu}\right)^{2}}
$$

and:

$$
\mu(\omega)=\sum_{\nu=1}^{N} \frac{\kappa_{\nu} \omega / \Omega_{\nu}}{1+\left(\omega / \Omega_{\nu}\right)^{2}}
$$

Nachman et al. (1990) state that these equations satisfy the Kramers-Kronig rclations.

\section{CONTINUUM OF RELAXATION PROCESSES}

\section{Three-parameter model}

In biological media, we often observe a nearly constant slope of the attenuation versus frequency over a large frequency range. If we assume that the attenuation slope is constant in a certain frequency band, then we can reduce the number of parameters considerably. This reduction is highly desirable from the parameter estimation point of view. If the relaxation frequencies are within half a decade apart, then the individual relaxation processes cannot be properly distinguished anymore. In that case, the sensitivity to one of the relaxation processes becomes rather small. An additional problem is the availability of accurate absorption curves, which limits the usefulness of a large number of parameters for description of our tissue model. Therefore, we will assume a continuous distribution of relaxation processes with analytical descriptions of density and relaxation strength over the frequency range of interest. To obtain the desired behaviour, we assume that the relaxation frequencies are distributed logarithmically over the frequency band. This can be stated as follows:

$$
\Omega_{\nu}=\Omega_{0} e^{\beta \nu}, \quad \nu=1, \ldots, N
$$

where $\Omega_{0}$ is a reference frequency and $\beta$ is a constant prescribing a fixed log-frequency distance between the relaxation processes. Furthermore, we assume that the value of $\kappa_{\nu}$ is a constant with value $\kappa_{r e l}$. The angular frequency band of interest is defined as $\omega_{L} \leq \Omega_{\nu} \leq$ $\omega_{H}$. Finally, we assume that the relaxation processes are a continuous distribution over the frequency range instead of a discrete sum. In view of the continuous form of eqn (12), viz. $\Omega(\nu)=\Omega_{0} e^{\beta \nu}$, the continuous equivalent of eqn (10) becomes:

$$
\lambda(\omega)=\frac{\kappa_{r e l}}{\beta} \int_{\omega_{L}}^{\omega_{H}} \frac{\Omega(\nu)}{\Omega^{2}(\nu)+\omega^{2}} d \Omega(\nu)
$$

which can be evaluated as:

$$
\lambda(\omega)=\frac{\kappa_{r e l}}{2 \beta} \log \left(\frac{\omega_{H}^{2}+\omega^{2}}{\omega_{L}^{2}+\omega^{2}}\right)
$$

We convert the discrete form of $\mu(\omega)$ to a continuous form in a similar way. Using eqn (12), eqn (11) can be written as:

$$
\mu(\omega)=\frac{\kappa_{r e l}}{\beta} \int_{\omega_{L}}^{\omega_{H}} \frac{\omega}{\Omega^{2}(\nu)+\omega^{2}} d \Omega(\nu)
$$

which can be evaluated as:

$$
\mu(\omega)=\frac{\kappa_{r e l}}{\beta}\left[\arctan \left(\omega / \omega_{H}\right)-\arctan \left(\omega / \omega_{L}\right)\right]
$$

Equations (14) and (16) can be used in eqns (8) and (9) to obtain the desired sound speed and the attenuation coefficient. The corresponding form of the highfrequency sound speed, $c_{\infty}$, is obtained from eqn (8):

$$
c_{\infty}=\left(\frac{1}{c_{0}^{2}}-\frac{\rho_{0} \kappa_{r e l}}{\beta} \log \left(\omega_{H} / \omega_{L}\right)\right)^{-1 / 2}
$$

and the high-frequency compressibility $\kappa_{\infty}$ is

$$
\kappa_{\infty}=\frac{1}{c_{0}^{2} \rho_{0}}-\frac{\kappa_{r e l}}{\beta} \log \left(\omega_{H} / \omega_{L}\right)
$$

The model now contains three parameters: the angular frequencies, $\omega_{L}$ and $\omega_{H}$, and the ratio, $\kappa_{r e l} / \beta$. If we have a measured curve of $\alpha(\omega)$ then these three parameters can be obtained from a model fit. 
Two-parameter model

A further simplification results from a high frequency approximation for $\omega_{H}$, i.e., $\omega_{H} \gg \omega$. Then, we get:

$$
\mu(\omega) \approx-\frac{\kappa_{r e l}}{\beta} \arctan \left(\omega / \omega_{L}\right)
$$

Instead of an approximation for $\lambda(\omega)$, we use an approximation for $\kappa_{\infty}+\lambda(\omega)$, which occurs in eqns (8) and $(9)$ :

$$
\kappa_{\infty}+\lambda(\omega) \approx \frac{1}{c_{0}^{2} \rho_{0}}+\frac{\kappa_{r e l}}{2 \beta} \log \left(\frac{\omega_{L}^{2}}{\omega_{L}^{2}+\omega^{2}}\right)
$$

The equations for $c(\omega)$ [eqn (8)] and $\alpha(\omega)$ [eqn (9) ], then consist of only two parameters, namely $\kappa_{r e l} / \beta$ and $\omega_{L}$.

Using eqns (19) and (20), we can see that the quantity $\alpha(\omega) / \omega$ approximates a constant value for high frequencies $\omega \gg \omega_{L}$. If we define:

$$
\alpha_{\infty}^{\prime}=\lim _{\omega \rightarrow \infty} \frac{\alpha(\omega)}{\omega}
$$

and calculate the limit we obtain:

$$
\alpha_{\infty}^{\prime}=\sqrt{\frac{1}{2}\left(-\frac{1}{c_{0}^{2}}+\sqrt{\left.\frac{1}{c_{0}^{4}}+\frac{\rho_{0}^{2} \pi^{2} \kappa_{r e l}^{2}}{4 \beta^{2}}\right)}\right.}
$$

From this equation, the ratio $\kappa_{r e l} / \beta$ can be solved. However, we will use the small absorption approximation, $c_{0} \alpha_{\infty}^{\prime} \ll 1$. Notice that for nearly all biological media this product is indeed very much smaller than unity, with a typical value of 0.001 . Applying the approximation, we obtain:

$$
\alpha_{\infty}^{\prime} \approx \frac{\pi}{4} \rho_{0} c_{0} \frac{\kappa_{r e l}}{\beta}, \quad c_{0} \alpha_{\infty}^{\prime} \ll 1
$$

Note that this equation does not impose further approximations to the relaxation model: it just gives a simple, approximate relation between the asymptote of the attenuation and one of the model parameters.

\section{Fit of two-parameter model to measured attenuation curve}

In this section, the two-parameter model will be fitted to the measured attenuation coefficient of Jongen et al. (1986). In that study, the attenuation coefficient of homogenized beef liver was measured, using two different transducers. Assuming a normal distribution of the measurement errors, the maximum likelihood estimate of the model parameters is obtained by minimizing the weighted-squared error (Bevington 1969)

$$
\chi^{2}=\sum_{i=1}^{N}\left(\frac{\alpha_{i}-\alpha\left(\omega_{i} ; \kappa_{r e l} / \beta, \omega_{L}\right)}{\sigma_{i}}\right)^{2}
$$

where $\alpha_{i}$ are the measured data at $\omega_{i}$ and $\alpha\left(\omega_{i} ; \kappa_{\text {rel }} l\right.$ $\left.\beta, \omega_{L}\right)$ are the predictions of the model at $\omega_{i}$, and $\sigma_{i}$ are the standard deviations of the measurement errors. The magnitude of $\sigma_{i}$ is $5 \%$ of the measured value. The model parameters, $\kappa_{r e l} / \beta$ and $\omega_{L}$, are obtained with a downhill simplex method (Grace 1992). In Fig. 1 we can see the fit obtained with the two-parameter model presented in this article. The resulting parameters are $f_{L}=3.22 \mathrm{MHz}$ and $\kappa_{\text {rel }} / \beta=1.033 \times 10^{-12}$. The latter parameter corresponds with a high-frequency attenuation slope, $\alpha_{\infty}^{\prime}=0.68 \mathrm{~dB} /(\mathrm{cm} \mathrm{MHz})$. The bars indicate the standard deviations of the measurement errors. The curve is quite similar to the curve obtained with the model of Jongen et al. (1986), which is also a continuous relaxation model. In fact, the normalized squared error is nearly the same for both methods, i.e., 0.030. However, the advantage of the present model is that an explicit expression is available for the dispersive sound speed $c$. For the power-law curve the error is 0.042 , i.e., somewhat larger than for our two-parameter model. A much larger error of 0.16 is obtained with a first-order polynomial. In Figs. 2 and 3, the results are shown for other medium properties which are derived from the fitted model parameters. The ambient sound speed is $c_{0}=1540 \mathrm{~m} / \mathrm{s}$, and the density equals $\rho_{0}=$ $10^{3} \mathrm{~kg} \mathrm{~m}^{-3}$.

\section{WAVE-FIELD COMPUTATIONS IN ABSORPTIVE MEDIA}

In this section, we show how the complex wavenumber, $k$, in the preceding section can be used efficiently to calculate images for transducers operating in pulse-echo mode. We employ an FFT-based k-space formulation of the wave-propagation problem. After Fourier-transforming the field at a certain depth, multiplication with a suitable $\mathbf{k}$-space Green's function and inverse Fourier transformation, the field at another depth can be obtained (Berkhout 1987). The necessary formulae are summarized in Appendix A. Discrete versions of the pertinent Fourier integrals can be computed efficiently with FFTs. However, the numerical implementation of the Fourier integrals is not trivial. If severe numerical enrors are to be avoided, we have carefully to implement the discretization of the spatial and spectral integrals, which is the subject of the following subsection. 


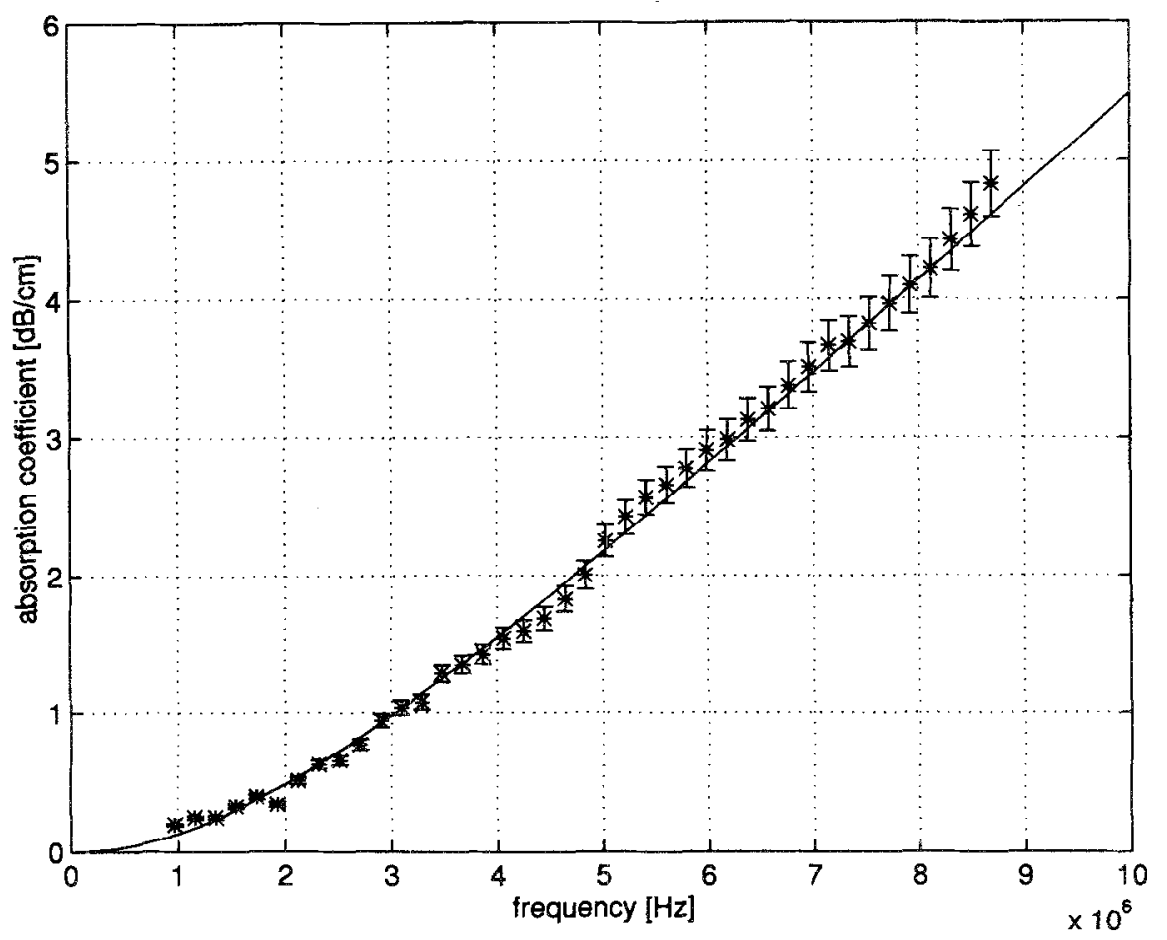

Fig. 1. Comparison of measured data of Jongen et al. (1986) to our two-parameter model for $f_{L}=\omega_{L} / 2 \pi=3.22$ $\mathrm{MHz}$ and $\kappa_{\text {rel }} l \beta=1.033 \times 10^{-12}$, i.e., $\alpha_{c c}^{\prime}-0.68 \mathrm{~dB} /(\mathrm{cm} \mathrm{MHz})$.
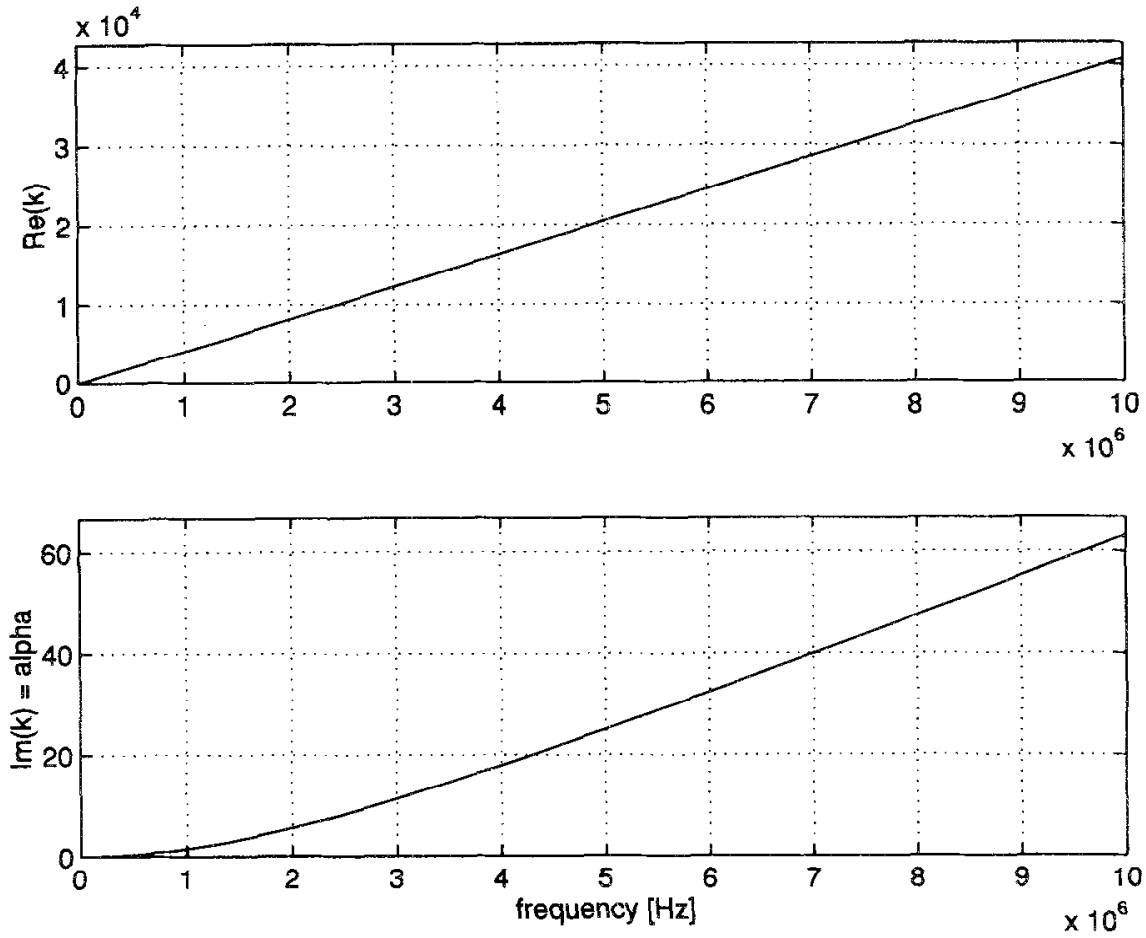

Fig. 2. Real and imaginary part of wavenumber $k(\omega)$ for the measured absorption curve. 


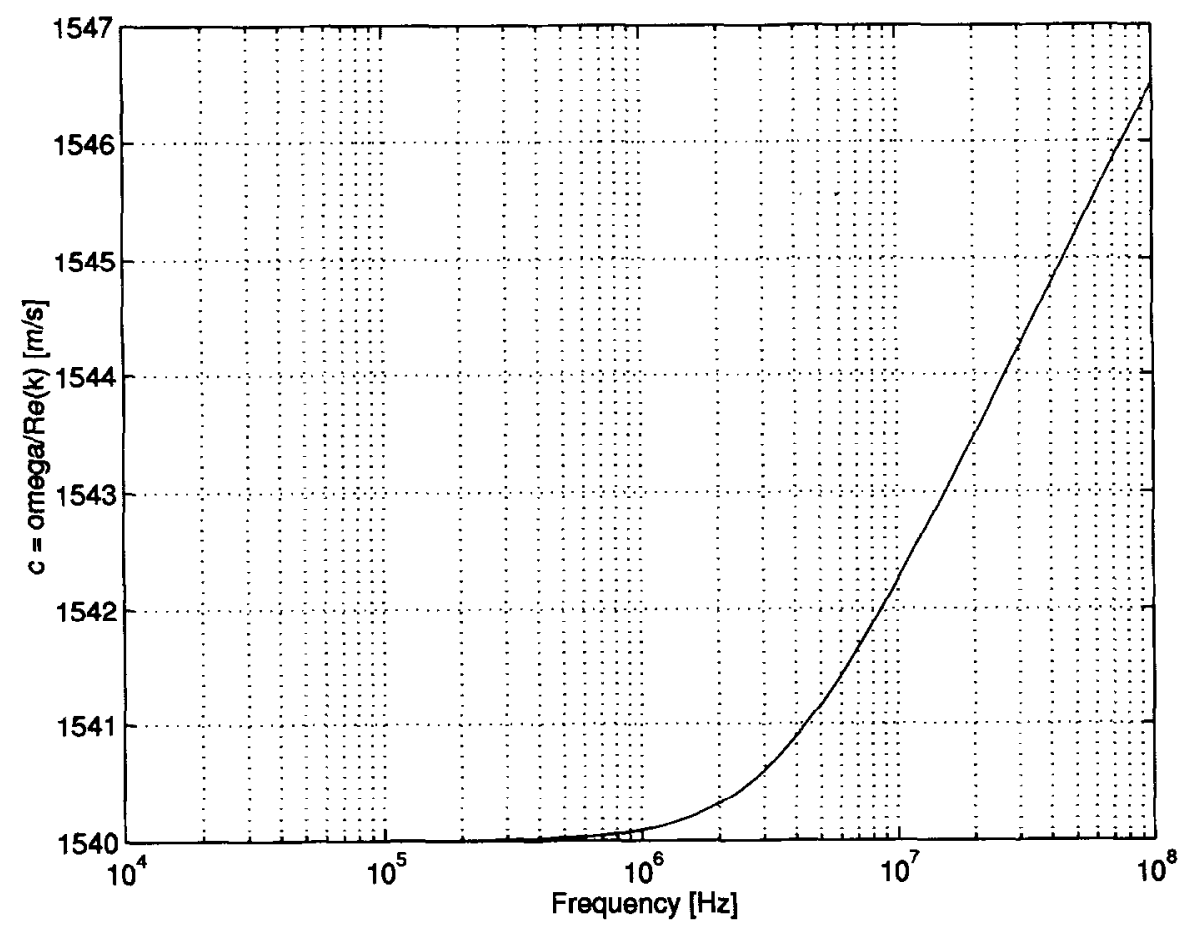

Fig. 3. Sound speed $c(\omega)$ for the measured absorption curve.

Weak forms of $\boldsymbol{k}$-space Green's functions

In several articles (Stepanishen and Benjamin 1982; Williams and Maynard 1982; Williams 1983) it was shown that planar acoustic wave-propagation problems can be solved very efficiently with FFTs. To avoid spatial aliasing, however, Williams and Maynard (1982) also showed that we cannot directly sample the k-domain Green's function. Accurate results are obtained if an averaged Green's function (Williams and Maynard 1982) is used. The averaged Green's function acts as a $\mathbf{k}$-space lowpass filter and reduces replicated sources and sampling errors. However, in the latter report, only the results were presented for extrapolation of normal velocity to pressure. Furthermore, formulae only were given for the limit of propagation over distance zero. In Appendix B, an extension is given for averaged Green's functions for normal velocity-to-pressure extrapolation, pressure-to-pressure extrapolation, and pressure-to-normal velocity extrapolation. The formulae are valid for arbitrary extrapolation distance $d$. This approach should also be contrasted to the approach of Pedersen and Orofino (1994) and Orofino and Pedersen (1993) where only the propagation between spectral pressure fields is described.

In Figs. 4-6 we can see the results for the spectral normal velocity to spectral pressure extrapolator, $\overline{\tilde{G}}_{v p}$, of eqns (B5) and (B6). In Fig. 4, the results are shown for the normal (sampled) Green's function and the averaged Green's function for $k d=10, \rho=\rho_{0}=$
$10^{3} \mathrm{~kg} \mathrm{~m}^{-3}$ and $f=\omega / 2 \pi=5 \mathrm{MHz}$. In Fig. 5, the results are shown for $k d=100$. It can be seen that the averaged function gradually attenuates the part of the Green's function where the slope of the phase curve becomes large. The differences between sampled and averaged Green's functions become larger for larger $k d$. In Fig. 6 , the results are shown for $k d=100$ with a fourfold increased sampling rate as compared to Fig. 5 . The transition point of attenuating the Green's function is moved toward a higher value of $k_{x} / k$. Therefore, sampling errors can be reduccd by increasing the sampling rate. The other two types of Green's functions, $\overline{\tilde{G}}_{p p}$ and $\overline{\tilde{G}}_{p v}$, show similar behaviour. However, the differences between the sampled and the averaged versions for the latter two Green's functions are not as large as for the first Green's function.

\section{IMAGING WITH A LINEAR ARRAY TRANSDUCER}

\section{Simulation configuration}

The configuration to be simulated is shown in Fig. 7. The array transducer consists of an array of 132 rectangular elements of infinite extent in the $y$-direction having a width of $0.3 \mathrm{~mm}$, and a distance (pitch) of $0.35 \mathrm{~mm}$ between the elements. We assume that the impulse response describing the transduction of element voltage to element surface velocity (and vice versa) is given by a modified Gaussian pulse, to be 

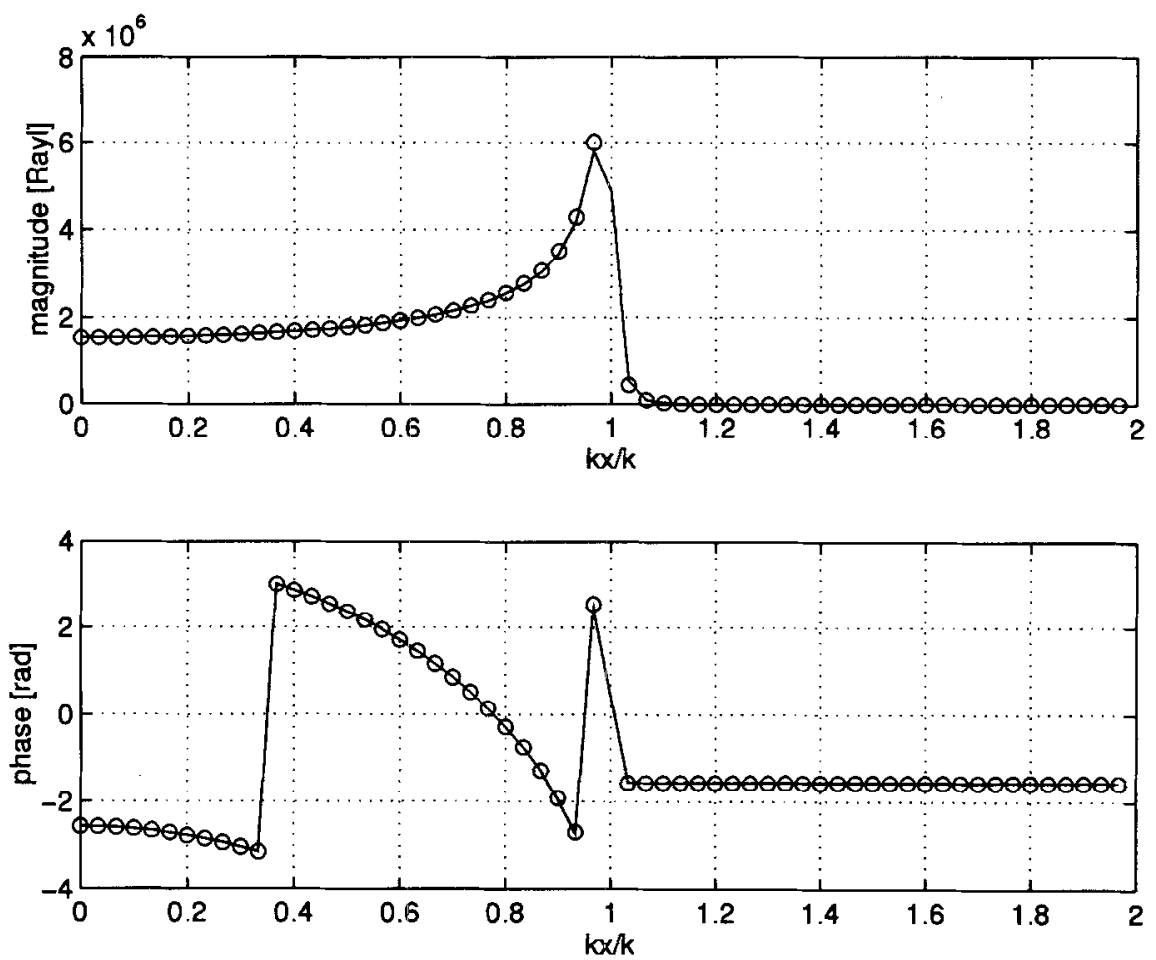

Fig. 4. Sampled Green's functions (small circles) and averaged Green's functions (solid line) for $k d=10$, velocity-to-pressure extrapolation.
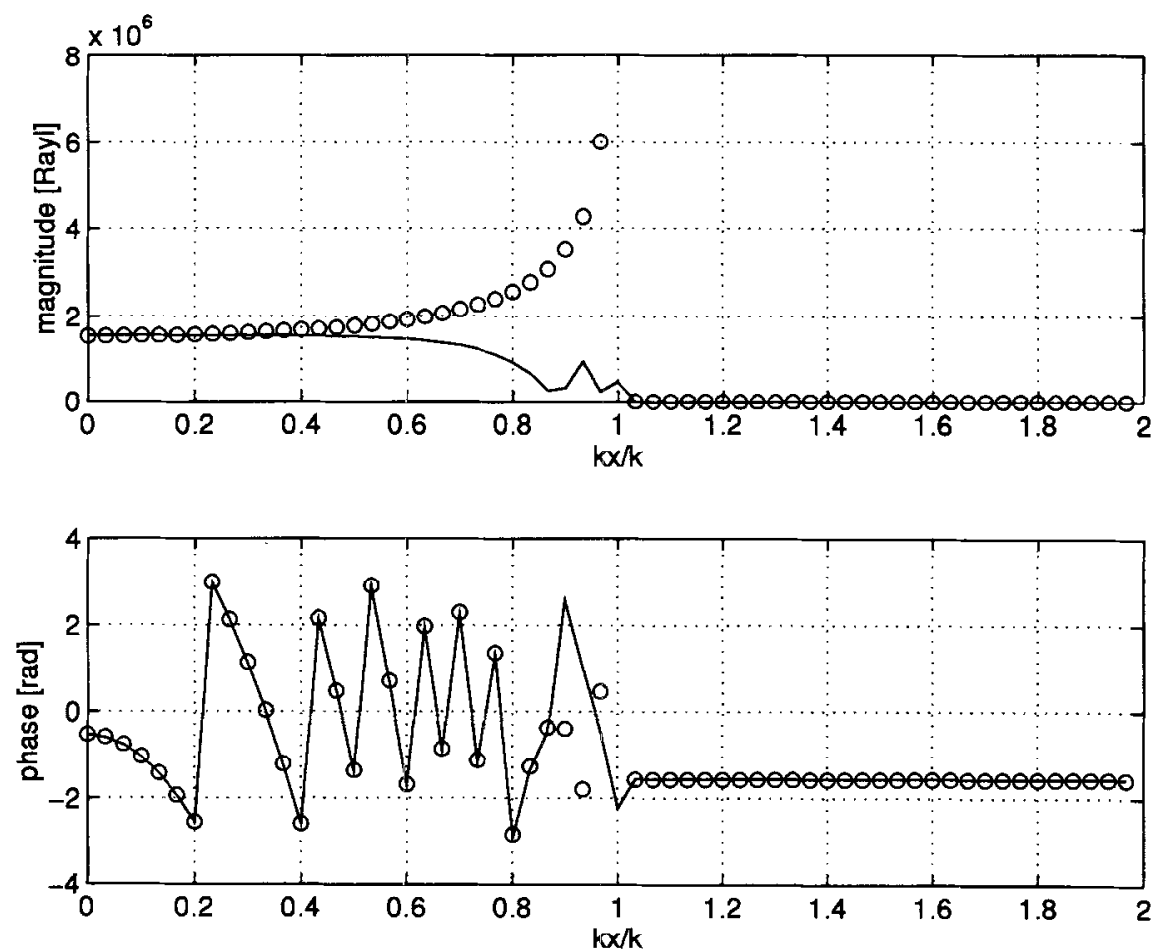

Fig. 5. Sampled Green's functions (small circles) and averaged Green's functions (solid line) for $k d=100$, velocity-tu-pressure extrapolation. 

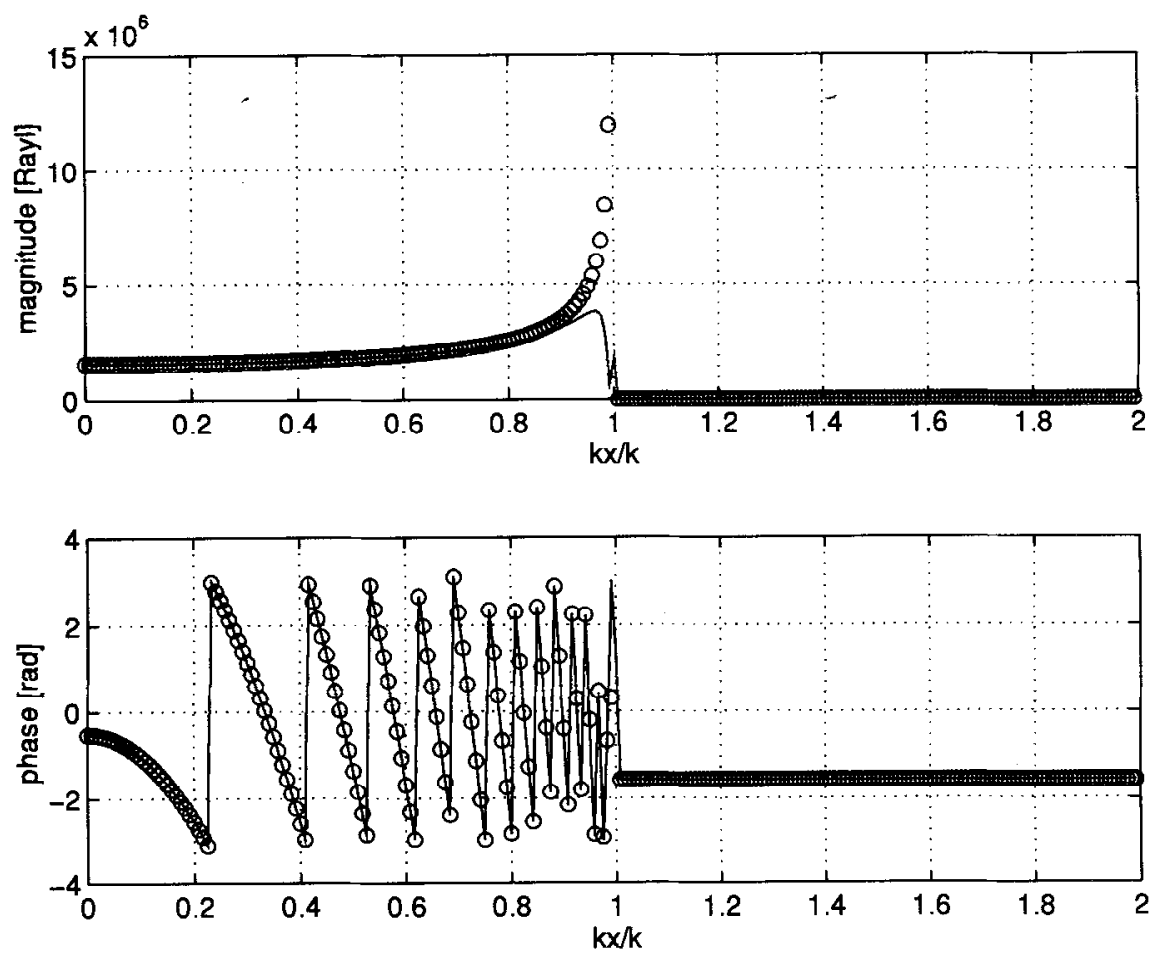

Fig. 6. Sampled Green's functions (small circles) and averaged Green's functions (solid line) for $k d=100$ with four times the sampling rate, velocity-to-pressure extrapolation.

described in the next subsection. The image is obtained by stepping a subset of 64 elements of the total of 132 elements along the array. Three line scatterers (points scatterers in two-dimensional space) are used simultaneously. The three scatterers are placed at the focal

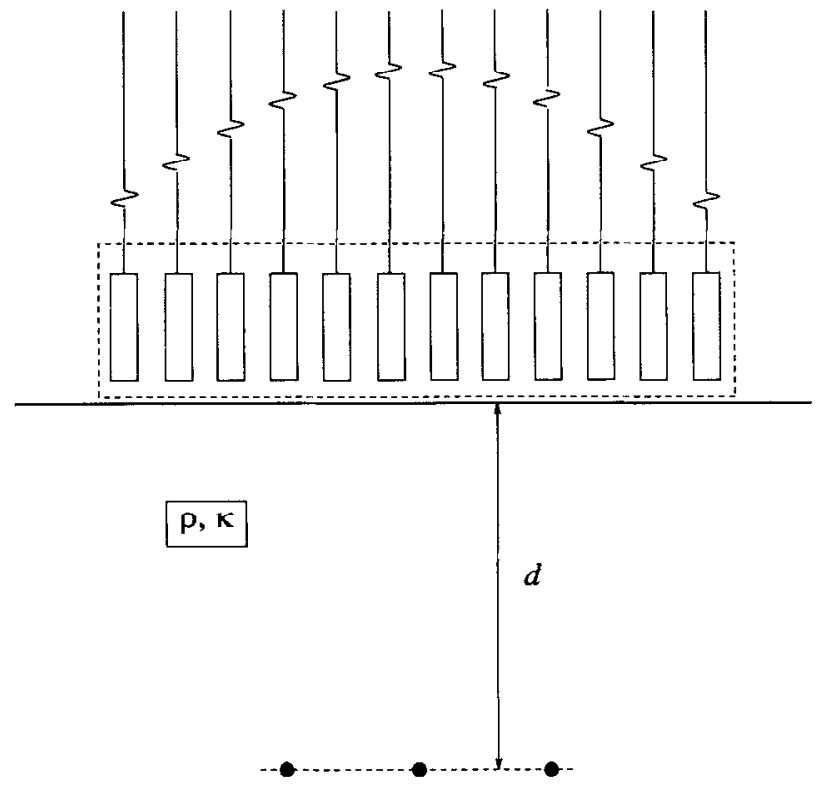

Fig. 7. Imaging configuration. distance, whereas the separation in the lateral direction is $0.01 \mathrm{~m}$. The absorptive medium consists of an infinite series of relaxation processes with parameters $f_{L}=$ $\omega_{L} /(2 \pi)=3.22 \mathrm{MHz}$ and a high-frequency attenuation slope of $\alpha_{\infty}^{\prime}-0.68 \mathrm{~dB} /(\mathrm{cm} \mathrm{MHz})$. In addition, simulations were performed for a medium without absorption and for an absorptive, dispersionless medium having the same attenuation $\alpha(\omega)$ as before, but where the sound speed $c(\omega)$ was assumed to be a constant $c=$ $1540 \mathrm{~m} / \mathrm{s}$. In all cases, the image axes were obtained by using the zero-frequency sound speed of $c_{0}=1540$ $\mathrm{m} / \mathrm{s}$ for conversion between time coordinates and spatial coordinates. The present two-dimensional configuration can be simulated by putting $k_{y}=0$ in Appendix $B$, where the spatial Fourier integrals reduce from twodimensional to one-dimensional.

\section{Pulse shape}

Especially for large-bandwidth transducers, the often-used Gaussian pulse shape is no longer representative for real transducers. We assume that the impulse response, $h(t)$, describing the transduction of element voltage to element surface velocity (and vice versa) is given by a modified Gaussian pulse (see Fig. 8). This impulse response is obtained from the convolution $h(t)=h_{1}(t) * h_{2}(t)$ of a Gaussian pulse, $h_{1}(t)$, having a center frequency $f_{c}=5 \mathrm{MHz}$ and a -6 -dB bandwidth 

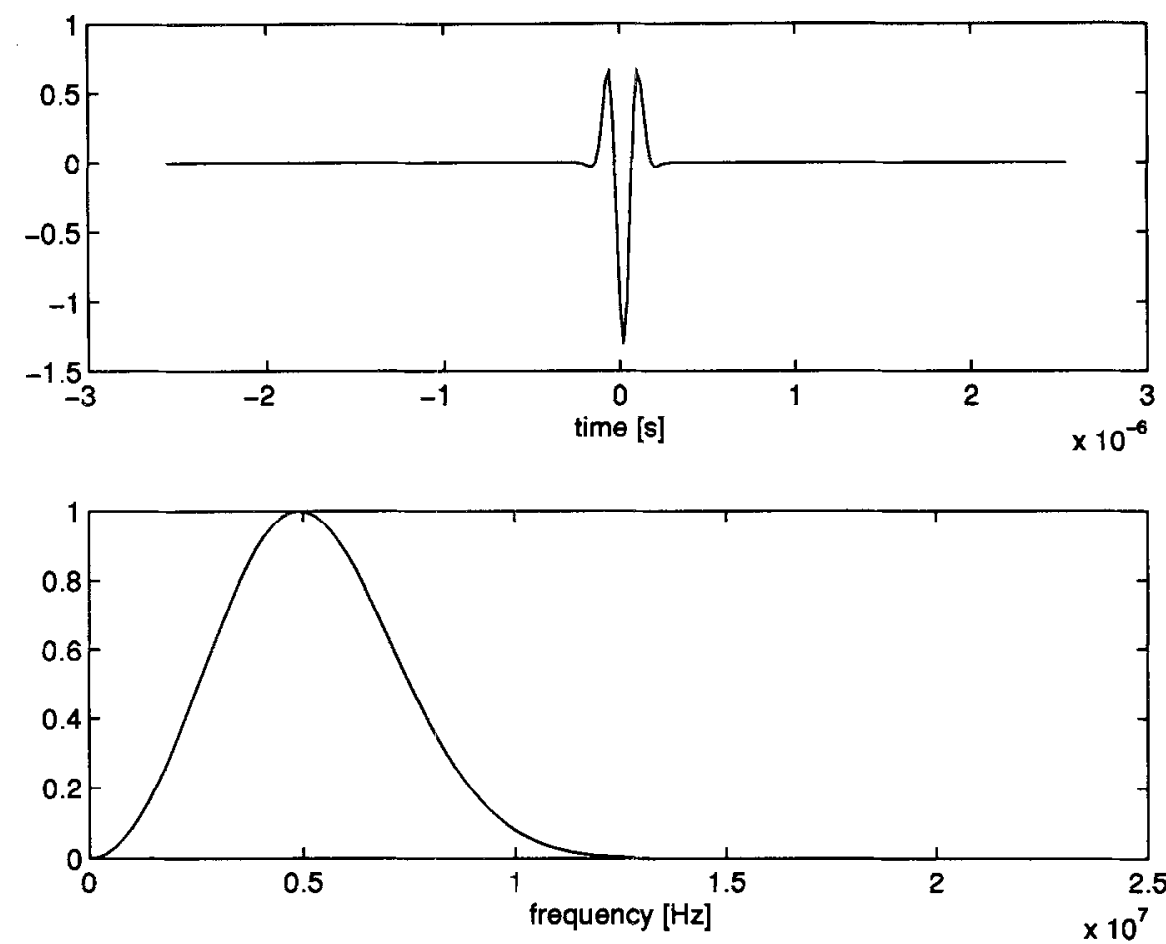

Fig. 8. Normal surface velocity of the central transducer elements in transmit mode.

of $1.75 f_{c}$ with pulse $h_{2}(t)$, as described by Berkhout (1987): $h_{2}(t)=\sqrt{\pi} f_{c}\left(0.5-\left(\pi f_{c} t\right)^{2}\right) \exp \left(-\left(\pi f_{c} t\right)^{2}\right)$. The advantage of this pulse $h(t)$ as compared to model-based pulses obtained with, for instance, the KLM model, is that we need only two parameters to describe our pulse shape. In our opinion, the use of this modified Gaussian is a useful starting point and reference for other pulse shapes.

\section{Transducer in transmit mode}

The starting point is a pulse voltage of specified amplitude and width. Delayed versions of this pulse are applied to each transducer element in such a way that the prescribed geometrical focus is obtained. The voltages of each transducer element are multiplied with the transducer characteristics in the frequency domain in order to obtain the normal element velocity. The transducer is assumed to have a uniform normal velocity for each element. The conversion of transducer velocity to a spatial sampling grid is obtained by linear interpolation, as done by Orofino and Pedersen (1993). In this way, we get a normal velocity distribution in the transducer plane. This normal velocity distribution is transformed (by spatial FFT) to the wavenumber domain. The sound field extrapolation to the scatterer plane results from the use of the velocity to pressure extrapolator of equs (B5) and (B6). To reduce the necessary length of the temporal FFTs, we used a phase correction to compensate for the estimated propagation time. This correction method has the advantage that the waveforms remain relatively short. As a consequence, the number of frequencies at which we have to analyze the complete problem is reduced considerably.

\section{Scaltering}

In the scatterer plane, we return to spatial coordinates and apply the scattcrer characteristics, in this case a filtered spatial and temporal Dirac function. After a spatial Fourier transform in the scatterer plane, use of the pressure to normal velocity propagation operator of eqns (B11) and (B12) and an inverse spatial Fourier transform, we obtain the normal velocity distribution in the transducer plane. As with the extrapolation from the transducer plane to the scattering plane, we applied a phase compensation to reduce the length of the signals in time. Notice that the computation time does not, in the first approximation, depend on the number of scatterers in the scatterer plane.

\section{Transducer in receive mode}

The normal velocity in the transducer plane is converted to an averaged normal velocity of each transducer element, again by linear interpolation. The element receive voltages are obtained by multiplication with the transducer characteristics in the frequency domain. The 

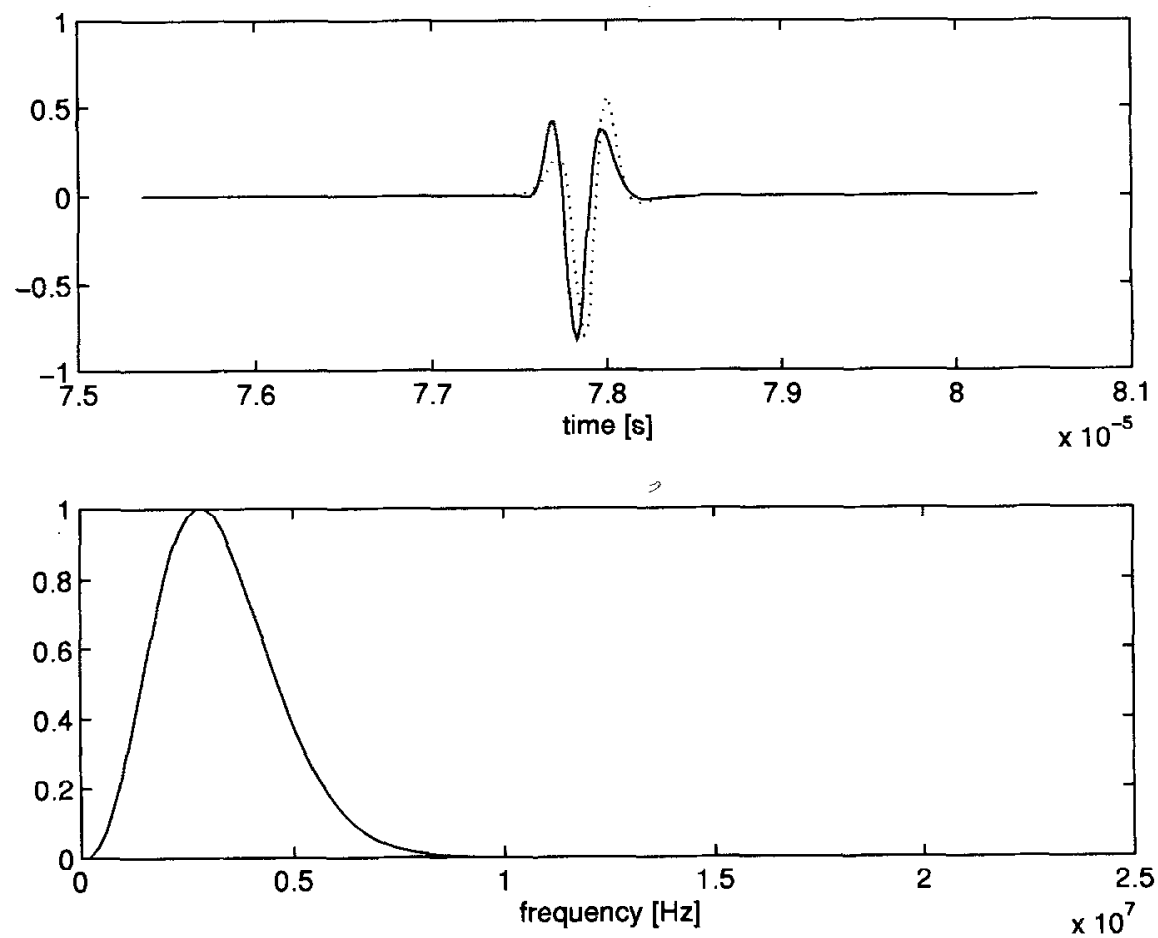

Fig. 9. Pressure in the focus at $z=0.12 \mathrm{~m}$, for the dispersive model (solid) and the nondispersive model (dotted).
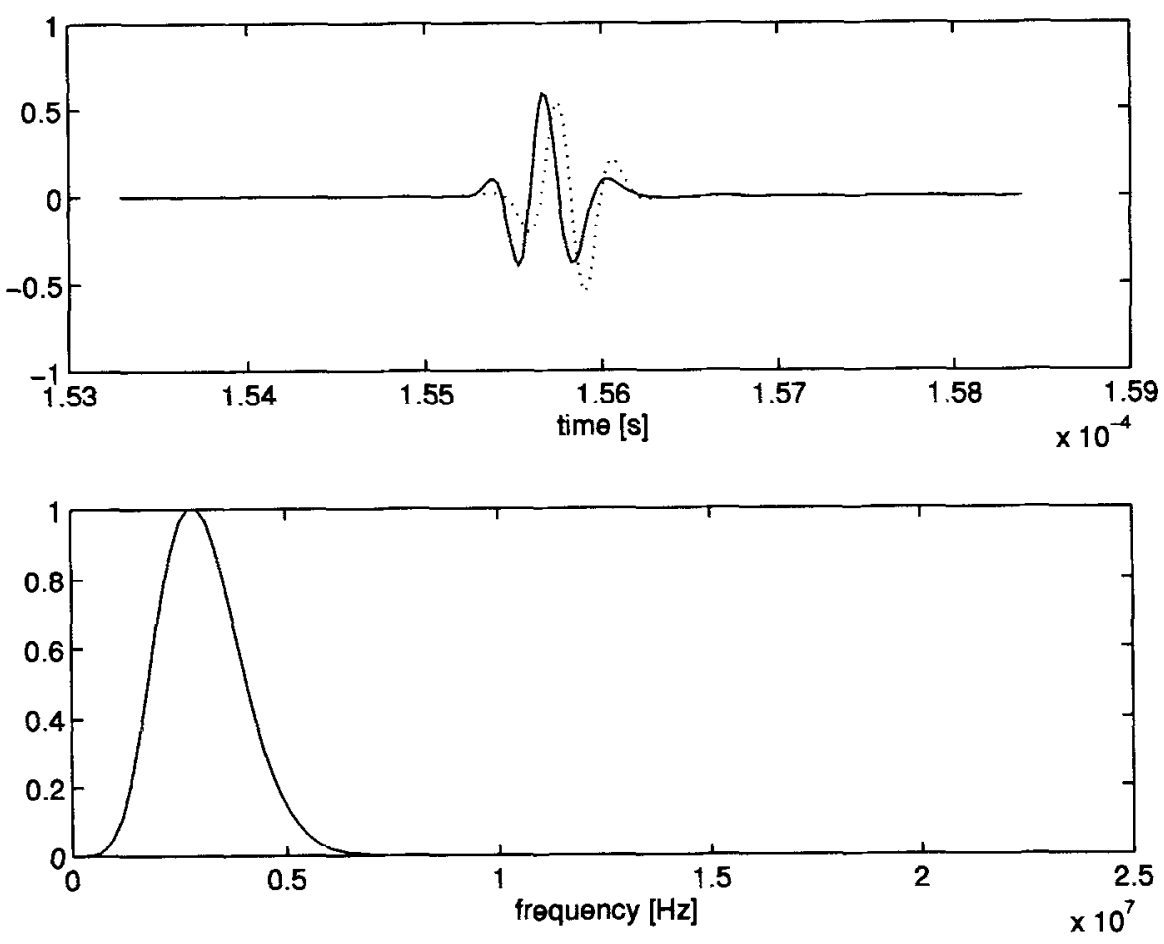

Fig. 10. Received RF signal from beamformer with focus and scatterers at $z=0.12 \mathrm{~m}$, for the dispersive model (snlid) and the nondispersive model (dotted). 


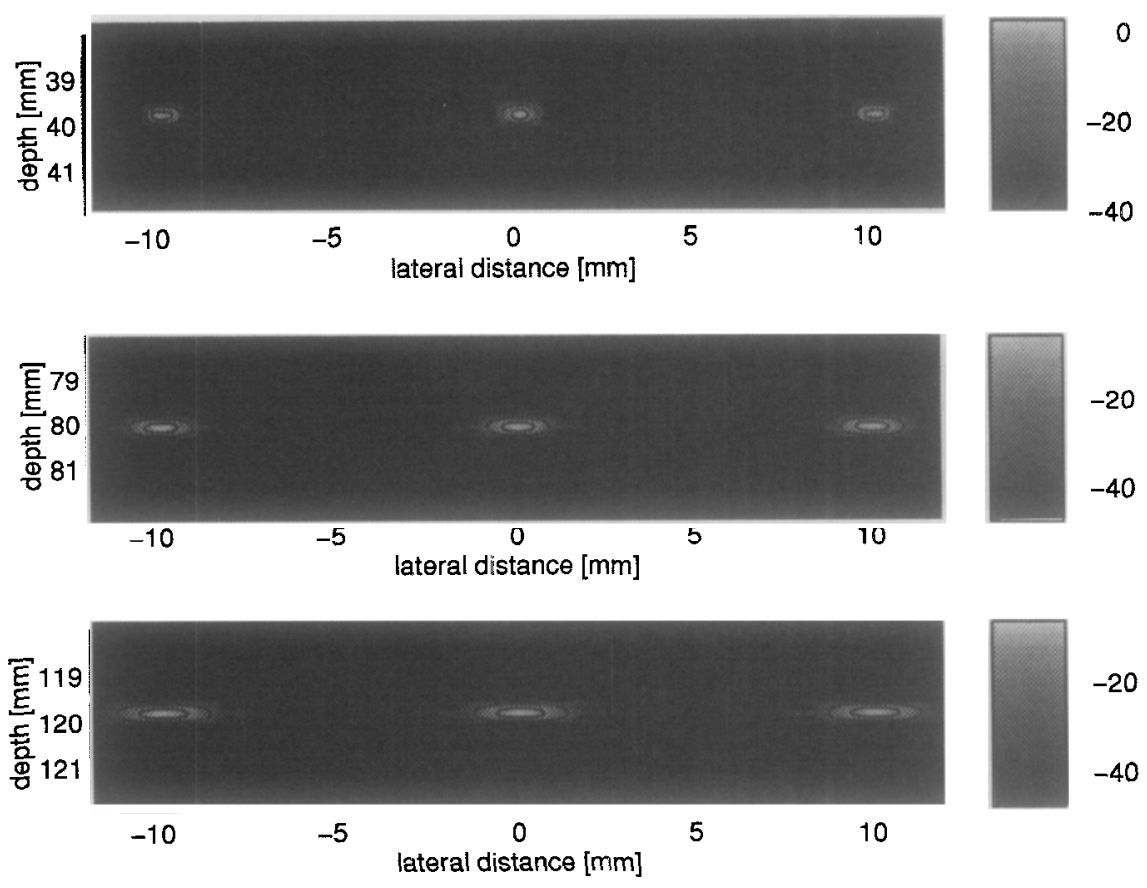

Fig. 11. Image for three line scatterers in a nonabsorptive medium, where the numbers to the right of the intensity bars denote the image amplitude in decibels; the image amplitudes are normalized with respect to the maximum of the upper image. Upper image: scatterers and focus at $z=40 \mathrm{~mm}$; middle image: scatterers and focus at $z=$ $80 \mathrm{~mm}$; lower image: scatterers and focus at $z=120 \mathrm{~mm}$.

output voltage of the receive beamformer, the RF signal, is obtained by summing delayed versions of the individual transducer element voltages. The same focal distance is used as for the transmit mode. For each scan position, the above procedure of transmitting and receiving is repeated. The final image is constructed by combining the
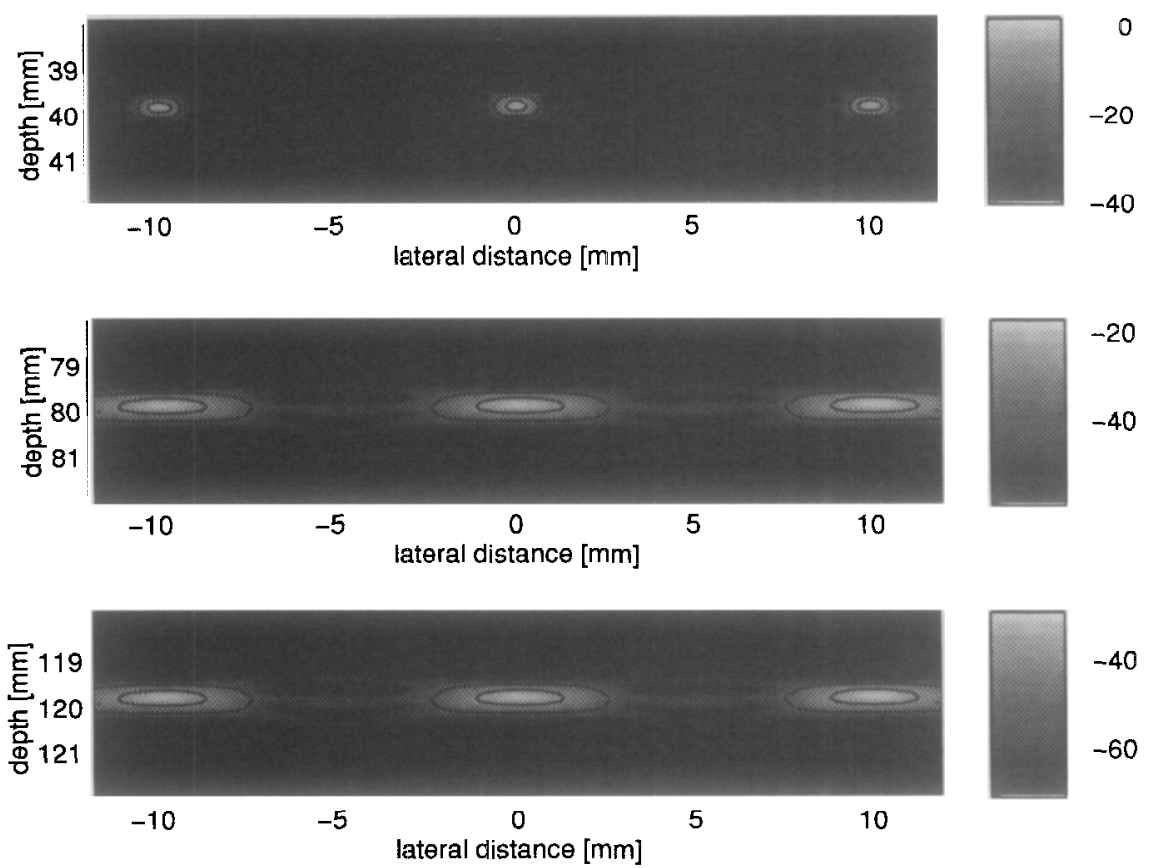

$-40$

$-60$

Fig. 12. As Fig. 11, except for the medium which has absorption characteristics described by the relaxation parameters $f_{L}=3.22 \mathrm{MHz}$ and $\alpha_{\infty}^{\prime}=0.68 \mathrm{~dB} /(\mathrm{cm} \mathrm{MHz})$. 

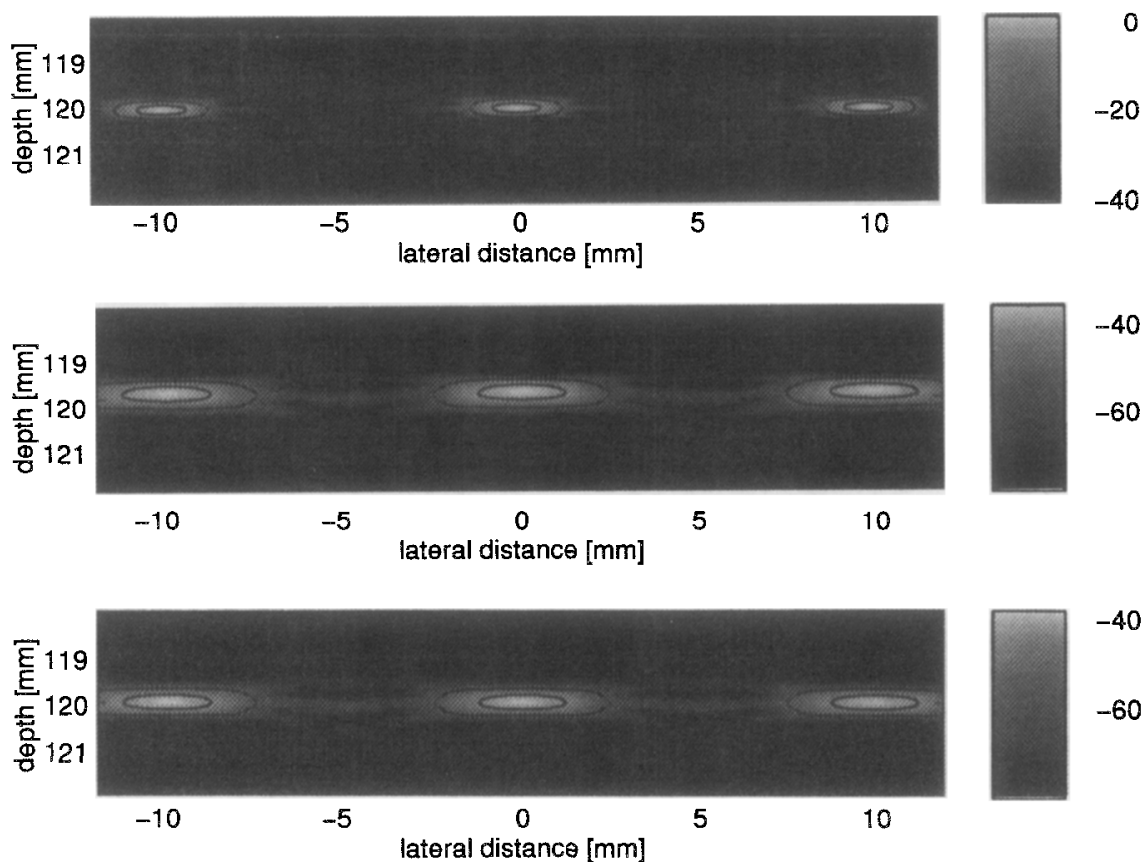

$-40$

$-60$

Fig. 13. Image for three line scatterers in an absorptive medium with scatterers and focus at $z=120 \mathrm{~mm}$, where the numbers to the right of the intensity bars denote the image amplitude in decibels; the image amplitudes are normalized with respect to the maximum of the upper image. Upper image: nonabsorptive medium; middle image: absorptive, dispersive medium with relaxation parameters $f_{L}=3.22 \mathrm{MHz}$ and $\alpha_{\infty}^{\prime}=0.68 \mathrm{~dB} /(\mathrm{cm} \mathrm{MHz})$; lower image: medium with same parameters as in the middle image with constant sound speed $c=1540 \mathrm{~m} / \mathrm{s}$ (i.e., without dispersion ).

envelope (by using a Hilbert transform) of each of the $69 \mathrm{RF}$ signals into a gray-scale image.

\section{Numerical parameters}

A spatial discretization step size, $\Delta x$, was chosen to be $\Delta x=0.25 \lambda$, with $\lambda$ the wavelength at a reference frequency. The reference frequency was chosen to be $7 \mathrm{MHz}$, i.e., somewhat above the central frequency of the transducer. The number of spatial points was 1024 . Due to the use of FFTs, the number of discrete plane waves was also 1024 . The temporal discretization was at a sampling rate of $50 \mathrm{MHz}$ at 256 discrete points in time. Some computation time was saved by rejecting the frequency components of the transducer pulse for which the magnitude was lower than 0.01 times the maximum value at $5 \mathrm{MHz}$.

Table 1. LSF characteristics of $z=40 \mathrm{~mm}$ for the $-20-\mathrm{dB}$ contour. Results are shown for medium with and without absorption.

\begin{tabular}{lllll}
\hline Medium & LSF $_{\mathrm{ax}}^{-20 \mathrm{~dB}}$ & $\mathrm{LSF}_{\mathrm{lat}}^{-20 \mathrm{~dB}}$ & $\mathrm{LSF}_{\text {area }}^{-20 \mathrm{~dB}}$ & LSF $_{\text {max }}$ \\
\hline Abs. & $0.42 \mathrm{~mm}$ & $1.2 \mathrm{~mm}$ & $0.44(\mathrm{~mm})^{2}$ & 0.14 \\
No abs. & $0.36 \mathrm{~mm}$ & $0.98 \mathrm{~mm}$ & $0.30(\mathrm{~mm})^{2}$ & 1 \\
\hline
\end{tabular}

\section{Numerical checks}

The calculations were checked for consistency by increasing the number of frequencies from 256 to 512 and by increasing the number of spatial samples from 1024 to 2048. In both cases, there was no significant difference in the images. The medium parameters were checked with the expected frequency shift of a Gaussian pulse in a medium with linear attenuation (Dines and Kak 1979) of $0.5 \mathrm{~dB} /(\mathrm{cm} \mathrm{MHz})$. The frequency shift for a central frequency of $3.5 \mathrm{MHz}$ and a bandwidth of $1.4 \mathrm{MHz}$ at $z=0.12 \mathrm{~m}$ (one-way) should be $3.9 \times 10^{5} \mathrm{~Hz}$ in such a medium. The simulations predicted a shift of $3.7 \times 10^{5} \mathrm{~Hz}$. However, it should be noted that our medium model does not have an attenuation which is exactly linear with frequency. Especially at very low frequencies this is not the actual case.

\section{Results}

For the transducer response of Fig. 8, we can see the pressure waveform in the focus at $z=0.12 \mathrm{~m}$ in Figure 9. In Fig. 10, the corresponding electrical receive beamformer RF signal is shown. The upper figures show the pulse shape, whereas the lower figures show the magnitude of the frequency components. The solid lines are obtained by using the full dispersive 
Table 2. LSF characteristics of $z=80 \mathrm{~mm}$ for the $-20-\mathrm{dB}$ contour. Results are shown for medium with and without absorption.

\begin{tabular}{lcccc}
\hline Mcdium & LSF $_{\mathrm{ax}}^{-20 \mathrm{~dB}}$ & $\mathrm{LSF}_{\mathrm{lat}}^{-20 \mathrm{~dB}}$ & LSF $_{\mathrm{arca}}^{-20, \mathrm{AB}}$ & LSF $_{\max }$ \\
\hline Abs. & $0.50 \mathrm{~mm}$ & $2.8 \mathrm{~mm}$ & $1.2(\mathrm{~mm})^{2}$ & 0.032 \\
No abs. & $0.36 \mathrm{~mm}$ & $1.8 \mathrm{~mm}$ & $0.54(\mathrm{~mm})^{2}$ & 1 \\
\hline
\end{tabular}

model. The dotted line is obtained by using the nondispersive attenuation model for which the sound speed is not a function of frequency. We can see that the pulse shapes differ but that the frequency domain magnitudes are the same for the dispersive and the nondispersive model. In Fig. 11, the image is shown for the three line scatterers in a nonabsorptive medium at three different distances. The results for a dispersive absorptive medium are shown in Fig. 12. In Fig. 13, the effect of dispersion is shown for an imaging distance of 120 $\mathrm{mm}$. All nine images have a dynamic range of $40 \mathrm{~dB}$. The image of one line scatterer is defined as the line spread function (LSF) of the system. Most of the characteristic parameters were evaluated from the $-6-\mathrm{dB}$ and $-20-\mathrm{dB}$ LSF contours, which are shown in the images. In Tables $1-3$, the maxima of the LSF are shown as well as some other image characteristics. For the nonabsorbing case, we see that the axial LSF size, $\mathrm{LSF}_{\mathrm{ax}}^{-20 \mathrm{~dB}}$, is nearly independent of the depth, whereas the lateral LSF size, $\mathrm{LSF}_{\mathrm{lat}}^{-20 \mathrm{~dB}}$, is roughly proportional to the depth. In case of an absorbing medium, it can be seen that both the axial and lateral LSF sizes incease with depth. Furthermore, the axial and lateral LSF sizes are both larger than in the nonabsorbing case, which also leads to a significantly larger LSF area, $\mathbf{L S F}_{\text {area }}^{-20 \mathrm{~dB}}$. Note that this area is not just the produst of the LSF length and widh but is a real area where the curvature of the LSF has been taken into account. The differences in the LSFs for absorbing and nonabsorbing media become larger for larger depths, as might be expected. From the comparison of Fig. 12 (dispersive absorptive medium) with the result for the nonahsorptive medium, shown in Fig. 11, it can be seen that the depth location of the image maxima for absorptive media does not exactly correspond with the location of the line scatterers. In the lower image of

Table 3. LSF characteristics of $z=120 \mathrm{~mm}$ for the $-20-\mathrm{dB}$ contour. Results are shown for medium with and without absorption.

\begin{tabular}{lllll}
\hline Medium & $\mathrm{LSF}_{\mathrm{ax}}^{-20 \mathrm{~dB}}$ & $\mathrm{LSF}_{\mathrm{iat}}^{-20 \mathrm{~dB}}$ & $\mathrm{LSF}_{\mathrm{area}}^{-20 \mathrm{~dB}}$ & $\mathrm{LSF}_{\text {max }}$ \\
\hline Abs. & $0.60 \mathrm{~mm}$ & $4.4 \mathrm{~mm}$ & $2.5(\mathrm{~mm})^{2}$ & 0.011 \\
No abs. & $0.36 \mathrm{~mm}$ & $2.6 \mathrm{~mm}$ & $0.80(\mathrm{~mm})^{2}$ & 1 \\
\hline
\end{tabular}

Fig. 13, the result for the nondispersive model is shown. The scatterer locations are exactly at a depth of $z=0.12 \mathrm{~m}$ in this case. If we compare the images of the nondispersive and dispersive model in Fig. 13 we can conclude that, apart from the differences in the location of the scatterers in the image, the dispersive character of the wavenumber for this moderately absorbing medium has very little effect on the images. The same conclusion was arrived at for single-element transducers by Oosterveld (1990). For media with strong absorption, however, visible effects might be more clear.

\section{CONCLUSION}

We have presented rigorous methods for simulating the pulse-echo behaviour of ultrasonic devices in a homogeneous absorptive and causal dispersive medium. Our two-parameter relaxation model, which is obtained from our causal three parameter model, corresponds well to measured attenuation curves. It has been shown how the resulting complex wavenumber can be used numerically to evaluate the wave propagation in absorptive media. We have presented three weak forms of k-space Green's extrapolation functions, with inherent spatial antialiasing behaviour. These functions allow extrapolation between arbitrarily mixed spectral pressure and spectral velocity variables. Examples were given of the imaging of line scatterers irradiated by a linear array transducer operating in pulse-echo mode. It was shown that the absorption has a large influence on image quality. On the other hand, the dispersion in moderately absorptive biological media has a negligible effect on the image quality for linear array transducers. Therefore, our conclusion is that, for typical linear arrays and typical biological media, the image modification due to medium absorption is caused mainly hy the magnitude modification of the different frequency components, and not by pulse modification due to phase velocity differences.

Acknowledgements - This work has been supported by a grant from Philips Medical Systems BV, Best, The Netherlands, and by a grant from the Technological Science Branch of the Dutch Organization for Scientific Research, Project No. NGN 11.2427. We are grateful to the reviewers for their constructive comments.

\section{REFERENCES}

Bamber JC. Attenuation and absorption. In: Hill CR, ed. Physical principles of medical ultrasonics. Chichester: Ellis Horwood, 1986:118-199.

Berkhout AJ. Applied seismic wave theory. Amsterdam: Elsevier, 1987.

Bevington PR. Data reduction and error analysis for the physical sciences. New York: McGraw-Hill, 1969.

Christopher PT, Parker KJ. New approaches to the linear propagation of acoustic fields. J Acoust Soc Am 1991;90:507-521. 
Clemmow PC. The plane wave spectrum representation of electromagnetic fields. Oxford: Pergamon, 1966.

Dines $\mathrm{K} \Lambda$, Kak $\Lambda \mathrm{C}$. Uitrasonic attenuation tomography of soft tissues. Ultrason Imag 1979;1:16-33.

Dunn F, Edmonds PD, Fry WJ. Absorption and dispersion of ultrasound in biological media. In: Schwan HP, ed. Biological engineering (Vol 9). New York: McGraw-Hill, 1969:205-332.

Foster DR, Arditi M, Foster FS, Patterson MS, Hunt JW. Computer simulations of speckle in B-scan images. Ultrason Imag 1983;5:308-330.

Goodman JW. Introduction to Fourier optics. San Francisco: McGraw-Hill, 1968.

Goodsitt MM, Madsen EL, Zagzebski JA. A three dimensional model for generating the texture in $\mathbf{B}$-scan ultrasound images. Ultrason Imag 1983;5:253-279.

Grace A. Matlab optimization toolbox. Natick, MA: The Mathworks Inc, 1992.

Gurumurthy KV, Arthur RM. A dispersive model for the propagation of ultrasound in soft tissue. Ultrason Imag 1982;4:355-377.

Jongen HAH, Thijssen JM, van den Aarssen M, Verhoer WA. A general model for the absorption of ultrasound by biological tissues and experimental verification. J Acoust Soc Am 1986;79:535-540.

Kuc R. Modeling attenuation of soft tissue with a minimum-phase filter. Ultrason Imag 1984;6:24-36.

Lerch R, Landes H. Finite element modeling of the pulse-echo behaviour of ultrasound transducers. Proceedings of the IEEE UFFC Ultrasonics Symposium, 1-4 November 1994, Cannes 1994; 1021-1025.

Nachman AI, Smith JF, Waag RC. An equation for acoustic propagation in inhomogeneous media with relaxation losses. J Acoust Soc Am 1990;88:1584-1595.

O'Donnell M, Jaynes ET, Miller JG. General relationships between ultrasonic attenuation and dispersion. $J$ Acoust Soc Am 1978;63:1935-1937.

O'Donnell M, Jaynes ET, Miller JG. Kramers-Kronig relationship between ultrasonic attenuation and phase velocity. J Acoust Soc Am 1981;69:696-701.

Oosterveld BJ. PhD thesis. University of Nijmegen, Nijmegen. The Netherlands, 1990.

Ophir J, Jaeger PM. Spectral shifts of ultrasonic propagation through media with nonlinear dispersive attenuation. Ultrason Imag $1982 ; 4 ; 282-289$.

Orofino DP, Pedersen PC. Efficient angular spectrum decomposition for acoustic sources. Part I: Theory. Part II: Results. IEEE Trans Ultrason Ferroelect Freq Control 1993;40:238-257.

Pauly H, Schwan P. Mechanism of absorption of ultrasound in liver tissues. J Acoust Soc Am 1971;2:692-699.

Pedersen PC, Orofino DP. Modeling of received signals from finite reflectors in pulse-echo ultrasound. Proceedings of the IEEE UFFC Ultrasonics Symposium, 1-4 November 1994, Cannes (in press ); 1994; 1177-1181.

Sehgal CM, Greenleaf JM. Ultrasonic absorption and dispersion in biological media: A postulated model. J Acoust Soc Am 1982;72:1711-1718.

Stepanishen PR, Benjamin KC. Forward and backward projection of acoustic fields using FFT methods. J Acoust Soc Am 1982;71:803-812.

Tumbull DH, Lum PK, Kerr AT, Foster FS. Simulation of B-scan images from two-dimensional transducer arrays: Part I-Methods and quantitative contrast measurements. Ultrason Imag 1992; 14:323-343.

Wells PNT. Biomedical ultrasonics. London: Academic Press, 1977.

Williams EG. Numerical evaluation of the radiation from unbaffled, finite plates using the FFT. J Acoust Soc Am 1983;74:343-347.

Williams EG, Maynard JD. Numerical evaluation of the Rayleigh integral for planar radiators using the FFT. J Acoust Soc Am $1982 ; 72: 2020-2030$.

\section{APPENDIX A}

In this appendix we will summarize the formulas for Fourier-based wave-field extrapolation, which is described for electromagnetics by
Clemmow (1966) and Goodman (1968) and for acoustics by Berkhout (1987). Symbols without tilde denote $x$-domain (spatial) variables, with $\mathbf{x}=(x, y, z)$, whereas symbols with tilde denote $\mathbf{k}$-domain (spectral) variables, with $\mathbf{k}=\left(k_{x}, k_{y}, k_{\text {s }}\right)$. A time-dependence, $e^{-i \omega t}$, is assumed, as in eqn (5). The component of wave-propagation vector in $z$-direction is given by $k_{z}$. All waves are travelling in the positive $z-$ direction. The pressure variables are denoted by $P_{z}$ and $\tilde{P}_{z}$. The normal components of the velocity variables $\left\{\mathbf{V}_{z}, \tilde{\mathbf{V}}_{z}\right\}$ are given by $\left\{V_{z}, \tilde{V}_{z}\right\}$ $=\mathbf{n}_{v} \cdot\left\{\mathbf{V}_{z}, \tilde{\mathbf{V}}_{-}\right\}$, with $\mathbf{n}_{z}$ the unity vector pointing in the $z$-direction. For acoustic pressure and velocity variables, the subscript $z$ indicates their definition in the plane at depth $z$.

$$
\begin{aligned}
& \tilde{\mathbf{V}}_{z}=\tilde{P}_{z} \mathbf{k} / \rho \omega \\
& \check{V}_{z}=\check{P}_{z} k_{z} / \rho \omega \\
& \left\{\begin{array}{l}
k_{z}=\left(k^{2}-k_{x}^{2}\right)^{1 / 2} \\
\left\{P_{z}, \mathbf{V}_{z}\right\}=\int_{-\infty}^{\infty}\left\{\tilde{P}_{z}, \tilde{\mathbf{V}}_{z}\right\} e^{i k_{x} x} d k_{x} \quad(2 \mathrm{D}) \\
\left\{\tilde{P}_{z}, \tilde{\mathbf{V}}_{z}\right\}=\frac{1}{2 \pi} \int_{-\infty}^{\infty}\left\{P_{z}, \mathbf{V}_{z}\right\} e^{-i k_{i} x} d x
\end{array}\right. \\
& k_{z}=\left(k^{2}-k_{x}^{2}-k_{y}^{2}\right)^{1 / 2} \\
& \left\{P_{z}, \mathbf{V}_{z}\right\}=\iint_{-\infty}^{\infty}\left\{\tilde{P}_{z}, \tilde{\mathbf{V}}_{z}\right\} e^{i\left(k_{x} x+k_{y}\right)} d k_{x} d k_{y} \\
& \left\{\tilde{P}_{z}, \tilde{\mathbf{V}}_{z}\right\}=\frac{1}{4 \pi^{2}} \iint_{-\infty}^{\infty}\left\{P_{z}, \mathbf{V}_{z}\right\} e^{-i\left(k_{z} x+k_{y} y\right)} d x d y \\
& \left\{\tilde{\boldsymbol{P}}_{z_{2}}, \tilde{\mathbf{V}}_{z_{2}}\right\}=e^{i k\left(z_{2}-z_{1}\right)}\left\{\tilde{\boldsymbol{P}}_{z_{1}}, \tilde{\mathbf{V}}_{z_{1}}\right\}, \quad z_{2} \geq z_{1}
\end{aligned}
$$

Equations (A1) and (A2) relate the plane-wave decomposition of velocity to the plane-wave decomposition of the pressure at the same depth $z$. Equations (A3) and (A4) allow the transformation between plane-wave decomposition and spatial variables, both at depth $z$. Equation (A5) allows the extrapolation from the plane-wave decomposition at depth $z_{1}$ to the plane-wave decomposition at depth $z_{2}$.

\section{APPENDIX B}

In this appendix we show how the Fourier integrals in Appendix A can lead to an efficient and accurate FFT-based method for wavefield propagation. The Green's functions of this appendix allow us to propagate wave fields directly from a source plane to a target plane, irrespective of whether we deal with pressure or normal veloc ity variables. This approach should be contrasted with the approach of Orofino and Pedersen (1993) and Pedersen and Orofino (1994), where the complete propagation process is kept in spectral pressure variables. Therefore, in the case of a given transducer velocity and use of the methods of the aforementioned two articles, first an additional conversion has to be made between velocity and pressure variables at the transducer plane. In the following, we will show that these conversions are not necessary. To arrive at the desired results, we will derive weak forms for the three possible cases: pressure-to-pressure propagation, normal velocity-to-pressure propagation and pressure-to-normal velocity propagation.

\section{Sector average of $\boldsymbol{k}$-space extrapolators}

Numerical implementation of the formulae of Appendix A requires a discrete formulation of the continuous integrals. To reduce sampling errors, the integrands of the spectral integrals are multiplied with a weighting function and integrated over the $k_{x, v}$-plane. Use of a particular shifted version of this weighting function for each sampling point $m, n$ in $k_{x, v}$-space results in the discrete set of coefficients suitable for FFT transformation to the spatial domain. Analytical results can be obtained if we integrate over a sector in the $k_{x, y}$-plane. 
Relatively simple averaged Green's functions result if we use a constant box-shaped weighting function. Using this weighting function, the average $\widetilde{G}$ of the Green's function, $\widetilde{G}$, can be defined by:

$$
\overline{\bar{G}}=\frac{1}{(\Delta k)^{2}} \iint_{(\Delta k)^{2}} \tilde{G}\left(k_{x}, k_{y}, d\right) d k_{x} d k_{y}
$$

where $\Delta k$ is the stepsize in $k_{x, y}$ used in the FFT algorithms and $d$ $=z_{2}-z_{1}$ is the extrapolation distance. The averaging is more easily performed in polar coordinates, however. Then the averaging integral for the lattice point $\left(k_{x}, k_{y}\right)=(m \Delta k, n \Delta k)$ not coinciding with the spectral origin becomes:

$$
\overline{\tilde{G}}=\frac{1}{A} \int_{\phi_{1}}^{\phi_{\phi_{1}}+\Delta \phi} d \phi \int_{k_{0}-\wedge k / 2}^{k_{0}+\Delta k / 2} \tilde{G}\left(k_{x}, k_{y}, d\right) k_{\rho} d k_{\rho}, \quad m \neq 0 \cup n \neq 0
$$

where $k_{\rho}^{2}=k_{x}^{2}+k_{v}^{2}, k_{0}^{2}=\left(m^{2}+n^{2}\right)(\Delta k)^{2}$ and $A=\Delta \phi k_{0} \Delta k$ the area of the sector. The integrals are easily evaluated by making $k_{z}$ $=\left(k^{2}-k_{o}^{2}\right)^{1 / 2}$ the integration variable. We take $k_{1}=k_{0}-\Delta k / 2$, $k_{2}=k_{0}+\Delta k / 2$ and use $A=\Delta \phi\left(k_{2}^{2}-k_{1}^{2}\right) / 2$. For the lattice point at the spectral origin $\left(k_{x}, k_{y}\right)=(0,0)$, we use the slightly different form:

$$
\overline{\widetilde{G}}-\frac{1}{A_{0}} \int_{0}^{2 \pi} d \phi \int_{0}^{\Delta k / 2} \tilde{G}\left(k_{x}, k_{y}, d\right) k_{\rho} d k_{\rho}, \quad m, n-0
$$

where $A_{0}-\pi(\Delta k / 2)^{2}$.

\section{Analytical expressions for averaged Green's functions}

From eqns (A2) and (A5) we can see that the Green's function for spectral normal velocity to spectral pressure extrapolation is of the form:

$$
\tilde{G}_{v p}\left(k_{x}, k_{y}, d\right)=\rho \omega \frac{e^{i k d}}{k_{r}}
$$

Applying eqn (B2), and integrating, results in:

$$
\overline{\tilde{G}}_{v p}=2 i \rho \omega \frac{e^{i k_{z 2} d}-e^{i k_{1} d}}{\left(k_{2}^{2}-k_{1}^{2}\right) d}, \quad m \neq 0 \cup n \neq 0
$$

where $k_{11}=\left(k^{2}-k_{1}^{2}\right)^{1 / 2}$ and $k_{z 2}=\left(k^{2}-k_{2}^{2}\right)^{1 / 2}$. If the limit for $d \rightarrow$ 0 is taken, $\bar{G}_{u p}$ reduces to eqn (28) of Williams and Maynard (1982). The spectral zero-frequency component is obtained by applying eqn (B3):

$$
\overline{\tilde{G}}_{v p}=2 i \rho \omega \frac{e^{i k_{: \Delta} d}-e^{i k d}}{(\Delta k / 2)^{2} d}, \quad m . n=0
$$

where $k_{i \Delta}=\left(k^{2}-(\Delta k / 2)^{2}\right)^{1 / 2}$. Similarly, we can see from eqn (A5) that the Green's function for spectral pressure to spectral pressure extrapolation is of the form:

$$
\tilde{G}_{p p}\left(k_{x}, k_{y}, d\right)=e^{i k d}
$$

Applying eqn (B2) and integrating by parts results in:

$$
\overline{\tilde{G}}_{m p}=2 \frac{e^{i k_{z 2} d}\left[i k_{22} d-1\right]-e^{i k_{z 2} d}\left[i k_{z 1} d-1\right]}{\left(k_{2}^{2}-k_{1}^{2}\right) d^{2}}, \quad m \neq 0 \cup n \neq 0
$$

The spectral zero-frequency component is obtained from eqn (B3):

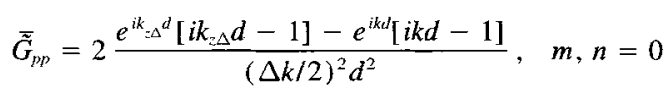

As before, eqns (A2) and (A5) show that the Green's function for spectral pressure to spectral normal velocity extrapolation is of the form:

$$
\tilde{G}_{m}\left(k_{x}, k_{y}, d\right)=\frac{1}{\rho \omega} k_{z} e^{i k_{z}^{d}}
$$

Applying eqn (B2) and integrating twice by parts results in:

$$
\begin{aligned}
& \overline{\tilde{G}}_{p v}=\frac{2 i}{\rho \omega\left(k_{2}^{2}-k_{1}^{2}\right) d^{3}}\left(e^{i k_{z 2} d}\left[k^{2} d^{2}-k_{2}^{2} d^{2}+2 i k_{z 2} d-2\right]\right. \\
& \left.-e^{i k_{1} d}\left[k^{2} d^{2}-k_{1}^{2} d^{2}+2 i k_{z 1} d-2\right]\right), \quad m \neq 0 \cup n \neq 0
\end{aligned}
$$

The spectral zero-frequency component is obtained from eqn (B3):

$$
\begin{array}{r}
\hat{G}_{p v}=\frac{2 i}{\rho \omega(\Delta k / 2)^{2} d^{3}}\left(e^{i k \Delta^{d}}\left[k^{2} d^{2}-(\Delta k / 2)^{2} d^{2}+2 i k_{z \Delta} d-2\right]\right. \\
\left.-e^{i k d}\left[k^{2} d^{2}+2 i k d-2\right]\right), \quad m, n=0 \quad
\end{array}
$$

Nyquist frequency for $\boldsymbol{k}$-space Green's functions

As a final result of Appendix B, an expression is derived that allows the computation of a Nyquist frequency for the $\mathbf{k}$-space Green's function. Above the Nyquist frequency, the discrete version does not uniquely represent the continuous version any longer. Our Nyquist frequency is defined as the spatial frequency where the phase difference between two samples becomes larger than $\pi$. If we write $\tilde{G}\left(k_{\rho}, d\right)=\tilde{G}\left(k_{x}, k_{y}, d\right)$, then the spatial Nyquist frequency, $k_{0}=k_{0, N_{v q}}$, can be defined with:

$\arg \left\{\tilde{G}\left(k_{0 . N y q}-\Delta k / 2, d\right)\right\}-\arg \left\{\tilde{G}\left(k_{0, N y q}+\Delta k / 2, d\right)\right\} \triangleq \pi$

For all three Green's functions given in the previous section this leads to

$$
\begin{aligned}
\left(k^{2}-k_{0, N y q}^{2}\right. & \left.+\Delta k k_{0, N y q}-(\Delta k / 2)^{2}\right)^{1 / 2} \\
& -\left(k^{7}-k_{0 . N y q}^{2}-\Delta k k_{0, N y q}-(\Delta k / 2)^{3}\right)^{1 / 2}=\frac{\pi}{d}
\end{aligned}
$$

This equation can be solved numerically. Keasonably good approximations and somewhat more insight can be obtained if we use the assumption of small sampling steps, i.e., $\Delta k / k \ll 1$. If also the assumption of a small Nyquist frequency is used, i.e., $k_{0 . N y q} / k \ll 1$, we get:

$$
k_{0, N y q} \approx \frac{\pi k}{\Delta k d}
$$

This formula shows that the Nyquist frequency is lower if the extrapolation distance $d$ is larger. This seems reasonable, since phase differences become larger for larger propagation distances. It can also be seen that the Nyquist frequency increases with decreasing normalized sampling distance, $\Delta k / k$. 